\title{
Mid-Term Results of Oxford Medial Unicompartmental Knee Arthroplasty in Young Asian Patients Less than 60 Years of Age: A Minimum 5-Year Follow-up
}

\author{
Yeong-Joon Kim, MD, Bu-Hwan Kim, MD, Seong-Ho Yoo, MD, Suk-Woong Kang, MD, Chang-Hun Kwack, \\ $\mathrm{MD}$, and Moo-Ho Song, MD \\ Department of Orthopaedic Surgery, Daedong General Hospital, Busan, Korea
}

\begin{abstract}
Purpose: The purpose of this study was to evaluate the efficacy of unicompartmental knee arthroplasty (UKA) in young active Asian patients by analyzing clinical outcomes, complications and survival rates.

Materials and Methods: Eighty-two knees were evaluated with a minimum follow-up of 5 years after Oxford phase 3 UKA in patients less than 60 years of age at the time of surgery. Their mean age was 54.7 years (range, 44 to 59 years). The mean follow-up period was 8.9 years (range, 5.3 to 12 years). Kaplan-Meier survivorship analysis was used to estimate implant survival.

Results: Including 3 bearing dislocations, 1 medial tibial collapse and 1 lateral osteoarthritis, the total complication rate was 6.1\% (5/82). Of the 3 cases of bearing dislocation, 2 cases were resolved by replacing with a thicker bearing and 1 case was converted to total knee arthroplasty (TKA) due to repeated dislocation. The two knees with a medial tibial collapse and a lateral osteoarthritis were converted to TKA. The 10-year cumulative survival rate using Kaplan-Meier survival method was $94.7 \%$ (95\% confidence interval: $88.7 \%-100 \%)$.

Conclusions: Oxford medial UKA was reliable and effective in young active Asian patients providing good clinical results and survival rate in the mid-term follow-up.
\end{abstract}

Keywords: Knee, Osteoarthritis, Arthroplasty, Unicompartmental, Mobile bearing, Asian, Young age

\section{Introduction}

Unicompartmantal knee arthroplasty (UKA) for the treatment of isolated compartment osteoarthritis of the knee has been continuously improved since its introduction in the 1950s. In 1989, Kozinn and $S \operatorname{cott}^{1)}$ reported the ideal candidates for UKA as inactive elderly ( $\geq 60$ years old) patients. Active patients and manual laborers were described as inappropriate candidates due to the

Received August 15, 2016; Revised (1st) October 7, 2016;

(2nd) November 9, 2016; Accepted December 20, 2016

Correspondence to: Moo-Ho Song, MD

Department of Orthopaedic Surgery, Daedong General Hospital, 187

Chungnyeol-daero, Dongnae-gu, Busan 47737, Korea

Tel: +82-451-554-8996, Fax: +82-451-553-7575

E-mail: mhsong21@hanmail.net

This is an Open Access article distributed under the terms of the Creative Commons Attribution Non-Commercial License (http://creativecommons.org/licenses/by-nc/4.0/) which permits unrestricted non-commercial use, distribution, and reproduction in any medium, provided the original work is properly cited. risk of mechanical failure and component loosening increased by repetitive high-impact loading. Thus, $<60$ years of age has been considered a contraindication for the procedure, and high tibial osteotomy (HTO) has been traditionally performed for relatively young patients. However, with advances in surgical instruments and techniques, a growing body of studies have demonstrated the outcomes of UKA in $<60$-year-old patients were comparable to those in $\geq 60$ years $^{2-4)}$. Furthermore, some studies have shown comparable or better results of UKA than HTO in young patients $^{5,6)}$.

However, it is disconcerting that young age appears to be associated with the increased risk of revision $\mathrm{UKA}^{7,8)}$. In particular, there are a number of studies suggesting polyethylene wear as a major mode of failure in fixed-bearing $\mathrm{UKA}^{9-12)}$. On the contrary, the use of a fully congruent mobile-bearing lowers contact stress, reducing the prospect of polyethylene wear and allowing for long-term follow-up of UKA ${ }^{13-15)}$. In this study, we retrospectively reviewed the mid-term results of minimally invasive Oxford me- 
dial UKA performed in young Asian patients ( $<60$ years of age at the time of surgery). The purpose of this study was to determine whether the procedure can be effective in young and active Asian patients based on the assessment of clinical outcomes, complications, and survivorship.

\section{Materials and Methods}

We obtained Institutional Review Board approval for retrospective data analysis. Between November 2003 and December 2010, 438 knees underwent medial UKA for the treatment of osteoarthritis. Of those, 82 knees in 78 patients $<60$ years of age and available for a minimum 5-year follow-up were included in this study. The age distribution of the total UKA patients (438 knees) was as follows: under 45 years, 1 (0.2\%); 45-49 years, 11 (2.5\%); 50-54 years, 42 (9.6\%); 55-59 years, 64 (14.6\%); 60-64 years, 85 (19.4\%); 65-69 years, 124 (28.4\%); 70-74 years, 81 (18.5\%); $75-79$ years, $29(6.6 \%)$; and over 80 years, $1(0.2 \%)$. Patients $<60$ years of age accounted for $26.9 \%$. Of the 78 patients ( 82 knees), 74 underwent unilateral UKA and 4 underwent bilateral UKA, and there were 75 female knees and 7 male knees. The mean follow-up period of the 82 knees was 8.9 years (range, 5.3 to 12 years): 5 years, 7 (8.54\%); 6 years, 3 (3.66\%); 7 years, 17 (20.73\%); 8 years, 10 (12.20\%); 9 years, 15 (18.29\%); 10 years, 21 (25.61\%); 11 years, $8(9.76 \%)$; and 12 years, $1(1.22 \%)$. Their mean age was 54.7 years (range, 44 to 59 years); mean height was $158 \mathrm{~cm}$ (range, 149 to $170 \mathrm{~cm}$ ); mean weight was $63.7 \mathrm{~kg}$ (range, 44 to $105 \mathrm{~kg}$ ), and mean body mass index was $25.5 \mathrm{~kg} / \mathrm{m}^{2}$ (range, 18.1 to 36.5 $\mathrm{kg} / \mathrm{m}^{2}$ ) (Table 1).

The clinical indication for surgery was pain in the medial aspect of the knee and the radiographic indication was Kellgren and Lawrence grade IV osteoarthritis. The patients' symptoms were refractory to prolonged conservative treatment, and thus knee replacement surgery was unavoidable. In all patients, the

Table 1. Dermographics

\begin{tabular}{lc}
\hline \multicolumn{1}{c}{ Characteristic } & Value \\
\hline No. of cases & 82 \\
Gender (female:male) & $75: 7$ \\
Follow-up (mo) & $106.7 \pm 20.6(63-144)$ \\
Age (yr) & $54.7 \pm 4.07(44-59)$ \\
Height $(\mathrm{cm})$ & $158.0 \pm 4.80(149-170)$ \\
Weight $(\mathrm{kg})$ & $63.7 \pm 8.64(44-105)$ \\
Body mass index $\left(\mathrm{kg} / \mathrm{m}^{2}\right)$ & $25.5 \pm 2.79(18.1-36.5)$ \\
\hline
\end{tabular}

Values are presented as mean \pm standard deviation (range). range of motion (ROM) was $\geq 110^{\circ}, \leq 10^{\circ}$ flexion contracture and $\leq 10^{\circ}$ varus deformity were observed, and the anterior and posterior cruciate ligaments were intact. Age and weight were not considered contraindications. Immediately before surgery, an arthroscopic examination was undertaken if necessary.

The surgery was performed by the same surgeon in all cases using the Oxford phase III (Biomet, Warsaw, IN, USA) prosthesis. Under spinal anesthesia, with a tourniquet applied to the upper thigh, the surgery was performed in a minimally invasive manner through a short longitudinal incision created on the medial aspect of the knee. Following cement fixation of the tibial and femoral components, a polyethylene insert was placed. Suction drainage was not inserted, and a compression bandage was applied after wound closure. Joint motion exercises and weightbearing ambulation with a walker was allowed from the 1st postoperative day.

Clinical assessment was performed preoperatively, 1 year after surgery, 2 years after surgery, and at the last follow-up using the ROM, Knee Society score (KSS), Western Ontario and McMaster Universities Osteoarthritis index (WOMAC). For radiological assessment, the tibiofemoral angle was measured on the weightbearing long leg radiograph, and component loosening or osteoarthritis in the other compartments was investigated on anteroposterior and lateral radiographs of the knee and patella

Statistical analysis was performed using IBM SPSS ver. 21 (IBM Co., Armonk, NY, USA). A paired $t$-test was used to compare pre- and postoperative differences in clinical assessment. A pvalue $<0.05$ was considered statistically significant. Kaplan-Meier survival analysis was performed to estimate the overall survival rate at 5,7 , and 10 postoperative years. Failure was defined as conversion to total knee arthroplasty (TKA) due to a complication.

\section{Results}

During the minimum 5-year follow-up of medial Oxford UKA in <60-year-old patients, complications occurred in $5(6.1 \%)$ of the total 82 knees: bearing in 3, medial tibial collapse in 1, and lateral osteoarthritis in 1 . Of the 3 bearing dislocation cases, replacement with a thicker bearing was done in 2 (Fig. 1) and the remaining 1 case was converted to TKA due to repeated dislocation. For medial tibial collapse (Fig. 2) and progressive lateral osteoarthritis (Fig. 3), conversion TKA was performed (Table 2). Other possible complications of UKA including infection, venous thromboembolism, ankylosis, and femoral/tibial component loosening were not observed. 

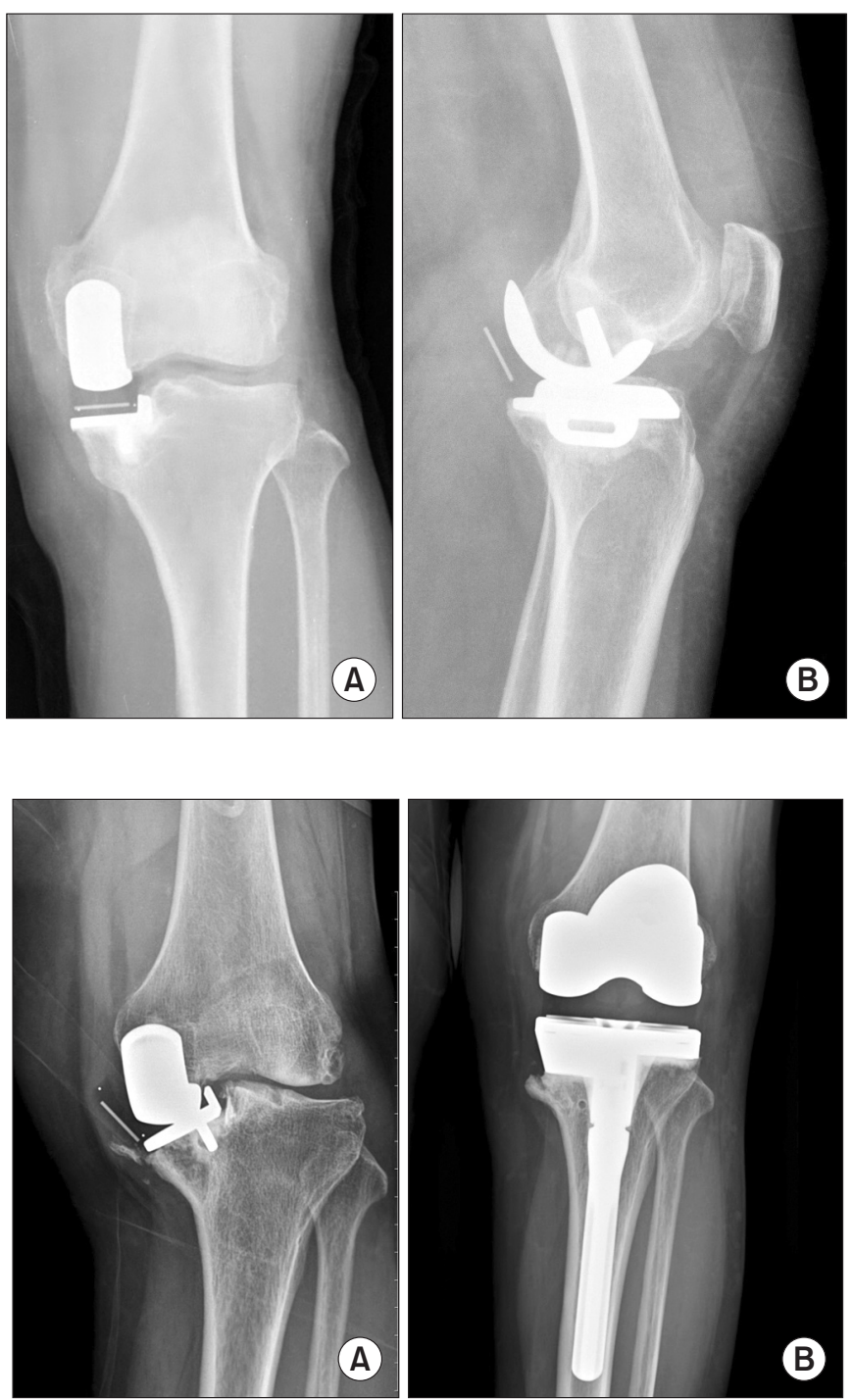

Fig. 2. (A) Medial tibial collapse of a 60-year-old female with a prior history of Oxford medial unicompartmental knee arthroplasty (7 years before the incident). (B) Conversion to total knee arthroplasty was performed with a metal block and a long tibial stem.

The mean KSS knee score was improved from 60.4 (range, 23 to 87 ) preoperatively to 94.2 (range, 68 to 100 ) postoperatively. The mean KSS function score increased from 51.7 (range, 20 to 90) preoperatively to 92.3 (range, 60 to 100) postoperatively. The mean preoperative WOMAC score was 58.4 (range, 34 to 87), which was statistically significantly improved to a mean of 10.8 (range, 0 to 28 ) postoperatively $(\mathrm{p}<0.001)$ (Table 3 ). However, there was no notable improvement in ROM: the preoperative mean value was $135.5^{\circ}$ (range, $120^{\circ}$ to $150^{\circ}$ ) and the last follow-up mean value was $137.9^{\circ}$ (range, $120^{\circ}$ to $\left.150^{\circ}\right)(\mathrm{p}=0.246)$ (Table 3 ).

Patient satisfaction grade according to the WOMAC score was fair in $31.7 \%$ and poor in $68.3 \%$ preoperatively. There was

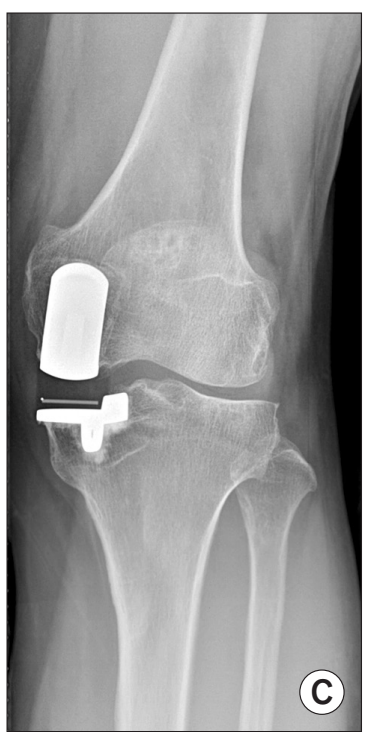

Fig. 1. (A) A 59-year-old female underwent Oxford medial unicompartmental knee arthroplasty 10 years ago. (B) Posterior dislocation of the meniscal bearing occurred 3 months after the operation. (C) She remained pain-free and obtained normal function of the left knee 9.8 years after bearing exchange.
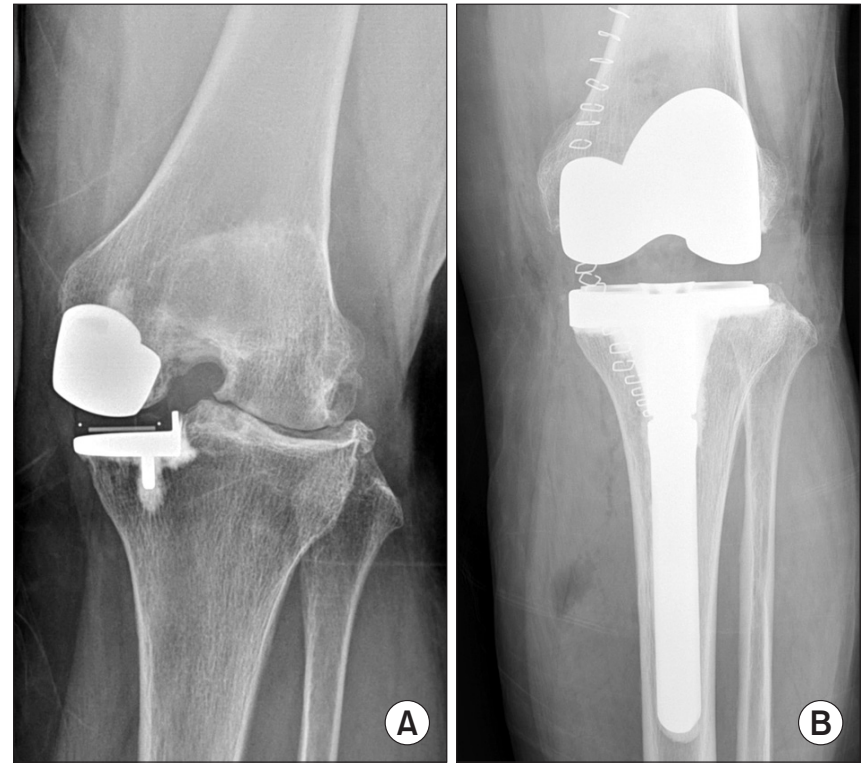

Fig. 3. (A) Progressive lateral osteoarthritis of a 65-year-old female with a previous history of Oxford medial unicompartmental knee arthroplasty (9.5 years previously). (B) Conversion to total knee arthroplasty was performed with a metal block and a long tibial stem.

a significant improvement at the last follow-up with excellent in $65.8 \%$, good in $29.3 \%$, fair in $4.9 \%$, and poor in $0 \%$. Patients with good or excellent scores were $95.1 \%$, indicating high patient satisfaction.

The mean femorotibial angle measured on the weight-bearing radiograph was $2.6^{\circ}$ varus (range, $9.1^{\circ}$ varus to $2.3^{\circ}$ valgus) preoperatively and $3.2^{\circ}$ of valgus (range, $2.2^{\circ}$ varus to $8.1^{\circ}$ valgus) at the last follow-up. There were no cases with radiographic evidence of $\geq 2 \mathrm{~mm}$ pathological radiolucency around the femoral/ 
Table 2. Details of 5 Cases with Complications

\begin{tabular}{clcll}
\hline Case & \multicolumn{1}{c}{ Complication } & Time to reoperation & \multicolumn{1}{c}{ Operative findings } & \multicolumn{1}{c}{ Procedure } \\
\hline 1 & Bearing dislocation & $3 \mathrm{mo}$ & MCL laxity & Bearing exchange, good result at 9 yr 10 mo \\
2 & Bearing dislocation & $1 \mathrm{yr} 4 \mathrm{mo}$ & MCL laxity & Bearing exchange, good result at 7 yr 4 mo \\
3 & Bearing dislocation & $2 \mathrm{yr} 6 \mathrm{mo}$ & Chronic MCL laxity & Conversion to TKA after recurrent bearing dislocation \\
4 & Medial joint collapse & $7 \mathrm{yr}$ & Medial tibial plateau fracture & Conversion to TKA with metal block and tibial stem \\
5 & Progressive lateral arthritis & $9 \mathrm{yr} 6 \mathrm{mo}$ & Lateral osteoarthritis & Conversion to TKA with metal block and tibial stem \\
\hline
\end{tabular}

MCL: medial collateral ligament, TKA: total knee arthroplasty.

Table 3. Clinical Results

\begin{tabular}{cccc}
\hline Variable & Preoperative & Postoperative & p-value \\
\hline Knee Society score & & & \\
Knee score & $60.4 \pm 18.46$ & $94.2 \pm 7.43$ & $<0.001$ \\
Function score & $51.7 \pm 10.89$ & $92.3 \pm 9.82$ & $<0.001$ \\
WOMAC (total) & $58.4 \pm 10.72$ & $10.8 \pm 7.04$ & $<0.001$ \\
Range of motion $\left(^{\circ}\right)$ & $135.5 \pm 5.66$ & $137.9 \pm 6.74$ & 0.246 \\
\hline
\end{tabular}

Values are presented as mean \pm standard deviation.

WOMAC: Western Ontario and McMaster Universities Osteoarthritis index.

tibial component or osteolysis. Although patellofemoral joint arthritis has been suspected in 2 cases, they are under close observation without any treatment due to the absence of symptoms. Three knees were found to have progressive arthritis in the lateral compartment, but only 1 knee with symptomatic arthritis was treated with TKA. On the Kaplan-Meier survival analysis, the median follow-up was 9.2 years, and the 5-year, 7-year, and 10year survival rates were $98.8 \%, 97.4 \%$, and $94.7 \%$, respectively. However, considering the small case number, the results should be interpreted with caution (Table 4 and Fig. 4).

\section{Discussion}

We followed 82 knees in 78 relatively young ( $<60$ years of age) Asian patients after Oxford UKA for a minimum of 5 years (mean, 8.9 years; range, 5.3 to 12 years). The significance of this study is that the 10-year survivorship of the implant with conversion to TKA as the endpoint was $94.7 \%$ in the Kaplan-Meier analysis. This finding is in disagreement with Kozinn and Scott ${ }^{1)}$ who reported that UKA was not an appropriate treatment option for $<60$-year-old young and active patients. There still is no consensus on the application of the procedure in young and active patients. Thompson et al. ${ }^{2)}$ and Pandit et al. ${ }^{4)}$ suggested that the $<60$-year-old contraindication should be modified. To our knowledge, however, mid-term results of UKA in $<60$-year-old
Table 4. Survival Rate at Each Time Point and 95\% Confidence Interval (CI)

\begin{tabular}{ccc}
\hline Set time $(\mathrm{yr})$ & Estimate $(\mathrm{SD})$ & $95 \% \mathrm{CI}$ \\
\hline 5 & $0.988(0.012)$ & $0.964-1.000$ \\
7 & $0.974(0.018)$ & $0.940-1.000$ \\
10 & $0.947(0.032)$ & $0.887-1.000$
\end{tabular}

SD: standard deviation.

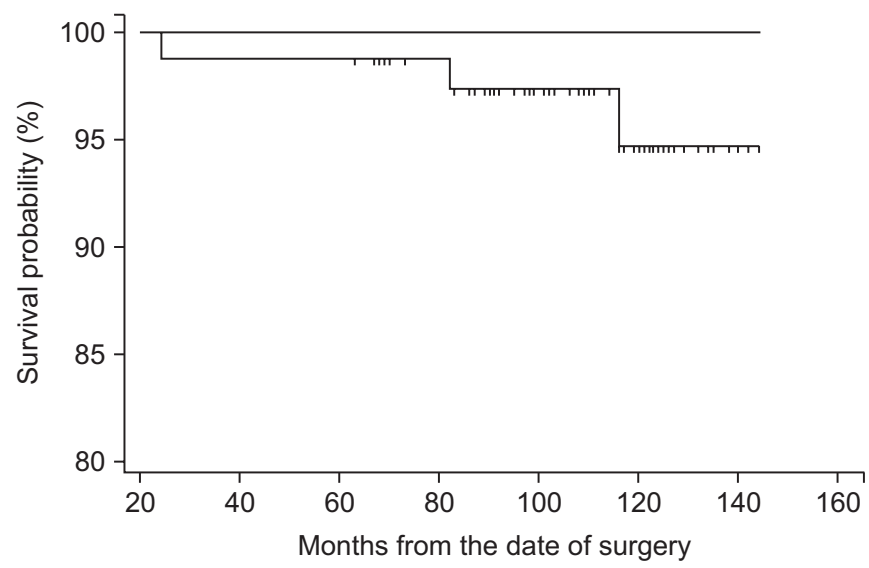

At risk (no.)

$\begin{array}{llllllll}82 & 81 & 81 & 73 & 49 & 27 & 2 & 0\end{array}$

Fig. 4. Kaplan-Meier survivorship curve with conversion to total knee arthroplasty as the endpoint. The cumulative survival rate is $94.7 \%$ at 10 years (95\% confidence interval: $88.7 \%-100.0 \%$ ).

Asian patients with a different lifestyle from Westerners have not been reported yet.

In the absence of gold standard treatments for medial unicompartmental arthritis in relatively young patients, TKA, UKA, and HTO have been considered the treatments of choice. In the past, TKA was most commonly performed among the three procedures. However, TKA has been associated with higher complication rates than $\mathrm{UKA}^{16)}$. In the meantime, clinical outcomes and long-term survivorship of UKA and HTO have been improved with advances in surgical instruments and techniques ${ }^{17-20)}$. Accordingly, the incidences of UKA and HTO are relatively increas- 
$\operatorname{ing}^{21,22)}$.

Traditionally, HTO has been the preferred option for the treatment of unicompartmental osteoarthritis of the knee in young and active patients; however, it can also lead to various complications $^{19,23)}$. Based on a review of the literature, Dettoni et al. ${ }^{5)}$ reported that 55 to 65 years old medial osteoarthritis patients were amenable to either HTO or UKA if they were non-obese and moderately active without joint instability and had mild varus deformity and good ROM. They also described that both procedures produced satisfactory outcomes with UKA offering slightly superior but statistically insignificant results in terms of clinical results and survivorship. Spahn et al. ${ }^{6}$ reviewed 5- to 8-year follow-up studies on HTO and UKA in a meta-analysis. They found that the two procedures were reliable and effective for medial osteoarthritis of the knee and had similar reoperation rates; however, clinical outcomes of UKA was superior to those of HTO. Recently, Petersen and Metzlaff ${ }^{24)}$ compared minimum 5 year follow-up results of the frequently used opening wedge HTO and mobile-bearing UKA performed in patients between 55 and 65 years of age. There was no significant difference in terms of reoperation rates; however, the incidence of residual pain and symptoms was higher after HTO, which they suggested patients should be informed of prior to surgery. Furthermore, they recommended UKA for patients expecting a higher quality of life.

The advantages of UKA include preservation of the intact weight-bearing compartment and anterior and posterior cruciate ligaments, which contributes to reducing intraoperative blood loss and postoperative pain for early rehabilitation, increasing the ROM, and causing low morbidity and mortality. In spite of such benefits, it has been contraindicated for $<60$ years of age since the report by Kozinn and Scott ${ }^{1)}$. However, recent studies have reported promising results in young patients, raising the question on the validity of the age-related contraindication ${ }^{2,4)}$. The 8 to 12 -year survival rates of UKA in $<60$-year-old patients range from $80 \%$ to $92 \%{ }^{3,9,25)}$. Still, it is discouraging to note that high levels of activity in young patients increase the risk of developing complications that eventually require a reoperation. W-Dahl et al. ${ }^{7)}$ reported that the reoperation rate was 3 times higher among patients $<55$ years of age than those $\geq 55$ years of age during a 10 year follow-up. Kuipers et al. ${ }^{8)}$ reported that patients $<60$ years of age had a 2.2-fold increased risk of revision when compared to those $\geq 60$ years of age. In particular, polyethylene wear has been frequently identified as the major cause of failure of fixed-bearing $\mathrm{UKA}^{9-12)}$. In contrast, the use of a fully congruent mobile-bearing lowers contact stress, reducing the prospect of polyethylene wear and thus allowing for a long-term follow-up of UKA ${ }^{13-15)}$. Howev- er, dislocation of the mobile-bearing may also occur, which puts the surgeons in dilemma. Bearing dislocation is the most common cause of failure that requires reoperation after UKA. The risk of bearing dislocation is 3 times higher in Asian patients than in their Western counterparts. Accordingly, mobile-bearing UKA may not be a proper solution for osteoarthritis in Asian patients living in cultures where high flexion of the knee is essential ${ }^{26)}$.

In our patients, bearing dislocation was also found to be the most common complication with an incidence of 3/82 (3.6\%), which is high compared to that in Western populations. Bearing dislocation should be considered as a potential complication for Asian patients undergoing mobile-bearing UKA considering their lifestyle that necessitates high flexion postures such as kneeling and squatting. The possible causes of bearing dislocation include inequality of the flexion and extension gaps, bearing impingement, abnormal positioning of the femoral or tibial component, chronic laxity of the medial collateral ligament, and habitual high flexion postures ${ }^{27)}$. If bearing dislocation occurs, replacement of the bearing, revision UKA, or conversion to TKA can be considered as possible treatment options. In a study by Lim et $\mathrm{al}^{28)}$, bearing dislocation was found in 12/400 knees (3\%) at a mean of 5.2 years after Oxford UKA. Of these 12 dislocation cases, 10 knees were treated by insertion of a thicker bearing, but conversion to TKA was required in only 2 knees. In our study, bearing dislocation occurred in 3 knees. Conversion to TKA was performed in only 1 knee for repeated dislocation. The remaining 2 knees exchanged with a thicker bearing has shown no further complication necessitating a revision surgery until the latest follow-up. Patient satisfaction at the last follow-up was good or excellent in 78 (95.1\%) of the total 82 knees except for 4 (fair, 4.9\%). According to Nwachukwu et al. ${ }^{21}$, both HTO and UKA can result in satisfying results if appropriate patients are selected for each procedure; however, there is a growing trend toward increased utilization of UKA while HTO is falling out of favor in the USA. They noted an overall 15-fold preference for UKA and it was more frequently performed in women and patients $>34$ years of age. This may be attributable to the fact that UKA allows for immediate weight-bearing after surgery and faster postoperative recovery. In contrast, HTO requires restrictions on weightbearing and a longer rehabilitation period. This process may be more difficult for older patients, and thus they prefer more convenient UKA. In addition, UKA is a cost-effective procedure because it necessitates shorter hospital stay and rehabilitation period and allows faster return to work than HTO. Furthermore, recent advancements in surgical instruments contributed to decreasing the learning curve of the once technically challenging 
procedure ${ }^{299}$. In spite of such advantages, Korean national registry data ${ }^{22)}$ shows that the utilization of HTO is more than twice that of UKA, contrary to the trend in the USA. In our opinion, this may not be because the outcomes of HTO are more satisfactory than those of UKA. Rather, it appears to be associated with economic reasons, such as reimbursement policies of the Health Insurance Review and Assessment Service in Korea.

Limitations of this study include the retrospective design and lack of comparison with $\geq 60$-year-old UKA patients or with fixed-bearing UKA in $<60$-year-old patients. However, the significance of this study is that mobile-bearing UKA showed promising results in $<60$-year-old Asian patients with a different lifestyle from Westerners in terms of the clinical improvement and longterm survivorship. Therefore, mobile-bearing UKA can be a viable alternative to the traditional HTO in young osteoarthritis patients where maintenance of activity levels and long-term survivorship are of prime importance in selecting surgical treatment.

\section{Conclusions}

Minimally invasive Oxford mobile-bearing UKA demonstrated excellent clinical improvement and survivorship in $<60$-year-old young Asian patients. Further follow-up should be performed to determine the long-term efficacy of the procedure.

\section{Conflict of Interest}

No potential conflict of interest relevant to this article was reported.

\section{References}

1. Kozinn SC, Scott R. Unicondylar knee arthroplasty. J Bone Joint Surg Am. 1989;71:145-50.

2. Thompson SA, Liabaud B, Nellans KW, Geller JA. Factors associated with poor outcomes following unicompartmental knee arthroplasty: redefining the "classic" indications for surgery. J Arthroplasty. 2013;28:1561-4.

3. Bruni D, Akkawi I, Iacono F, Raspugli GF, Gagliardi M, Nitri M, Grassi A, Zaffagnini S, Bignozzi S, Marcacci M. Minimum thickness of all-poly tibial component unicompartmental knee arthroplasty in patients younger than 60 years does not increase revision rate for aseptic loosening. Knee Surg Sports Traumatol Arthrosc. 2013;21:2462-7.

4. Pandit H, Jenkins C, Gill HS, Smith G, Price AJ, Dodd CA, Murray DW. Unnecessary contraindications for mobile- bearing unicompartmental knee replacement. J Bone Joint Surg Br. 2011;93:622-8.

5. Dettoni F, Bonasia DE, Castoldi F, Bruzzone M, Blonna D, Rossi R. High tibial osteotomy versus unicompartmental knee arthroplasty for medial compartment arthrosis of the knee: a review of the literature. Iowa Orthop J. 2010;30:13140.

6. Spahn G, Hofmann GO, von Engelhardt LV, Li M, Neubauer $\mathrm{H}$, Klinger HM. The impact of a high tibial valgus osteotomy and unicondylar medial arthroplasty on the treatment for knee osteoarthritis: a meta-analysis. Knee Surg Sports Traumatol Arthrosc. 2013;21:96-112.

7. W-Dahl A, Robertsson O, Lidgren L, Miller L, Davidson D, Graves S. Unicompartmental knee arthroplasty in patients aged less than 65. Acta Orthop. 2010;81:90-4.

8. Kuipers BM, Kollen BJ, Bots PC, Burger BJ, van Raay JJ, Tulp NJ, Verheyen CC. Factors associated with reduced early survival in the Oxford phase III medial unicompartment knee replacement. Knee. 2010;17:48-52.

9. Parratte S, Argenson JN, Pearce O, Pauly V, Auquier P, Aubaniac JM. Medial unicompartmental knee replacement in the under-50s. J Bone Joint Surg Br. 2009;91:351-6.

10. Koskinen E, Paavolainen P, Eskelinen A, Harilainen A, Sandelin J, Ylinen P, Tallroth K, Remes V. Medial unicompartmental knee arthroplasty with Miller-Galante II prosthesis: mid-term clinical and radiographic results. Arch Orthop Trauma Surg. 2009;129:617-24.

11. Ashraf T, Newman JH, Desai VV, Beard D, Nevelos JE. Polyethylene wear in a non-congruous unicompartmental knee replacement: a retrieval analysis. Knee. 2004;11:177-81.

12. Argenson JN, Parratte S. The unicompartmental knee: design and technical considerations in minimizing wear. Clin Orthop Relat Res. 2006;452:137-42.

13. Price AJ, Dodd CA, Svard UG, Murray DW. Oxford medial unicompartmental knee arthroplasty in patients younger and older than 60 years of age. J Bone Joint Surg Br. 2005;87:1488-92.

14. Streit MR, Streit J, Walker T, Bruckner T, Philippe Kretzer J, Ewerbeck V, Merle C, Aldinger PR, Gotterbarm T. Minimally invasive Oxford medial unicompartmental knee arthroplasty in young patients. Knee Surg Sports Traumatol Arthrosc. 2017;25:660-8.

15. Kendrick BJ, Simpson DJ, Kaptein BL, Valstar ER, Gill HS, Murray DW, Price AJ. Polyethylene wear of mobile-bearing unicompartmental knee replacement at 20 years. J Bone Joint Surg Br. 2011;93:470-5. 
16. Brown NM, Sheth NP, Davis K, Berend ME, Lombardi AV, Berend KR, Della Valle CJ. Total knee arthroplasty has higher postoperative morbidity than unicompartmental knee arthroplasty: a multicenter analysis. J Arthroplasty. 2012;27(8 Suppl):86-90.

17. Akizuki S, Shibakawa A, Takizawa T, Yamazaki I, Horiuchi H. The long-term outcome of high tibial osteotomy: a ten- to 20-year follow-up. J Bone Joint Surg Br. 2008;90:592-6.

18. Flecher X, Parratte S, Aubaniac JM, Argenson JN. A 12-28year followup study of closing wedge high tibial osteotomy. Clin Orthop Relat Res. 2006;452:91-6.

19. Fu D, Li G, Chen K, Zhao Y, Hua Y, Cai Z. Comparison of high tibial osteotomy and unicompartmental knee arthroplasty in the treatment of unicompartmental osteoarthritis: a meta-analysis. J Arthroplasty. 2013;28:759-65.

20. Heyse TJ, Khefacha A, Peersman G, Cartier P. Survivorship of UKA in the middle-aged. Knee. 2012;19:585-91.

21. Nwachukwu BU, McCormick FM, Schairer WW, Frank RM, Provencher MT, Roche MW. Unicompartmental knee arthroplasty versus high tibial osteotomy: United States practice patterns for the surgical treatment of unicompartmental arthritis. J Arthroplasty. 2014;29:1586-9.

22. Koh IJ, Kim MW, Kim JH, Han SY, In Y. Trends in high tibial osteotomy and knee arthroplasty utilizations and demographics in Korea from 2009 to 2013. J Arthroplasty. 2015;30:939-44.
23. Sun H, Zhou L, Li F, Duan J. comparison between closingwedge and opening-wedge high tibial osteotomy in patients with medial knee osteoarthritis: a systematic review and meta-analysis. J Knee Surg. 2017;30:158-65.

24. Petersen W, Metzlaff S. Open wedge high tibial osteotomy (HTO) versus mobile bearing unicondylar medial joint replacement: five years results. Arch Orthop Trauma Surg. 2016;136:983-9.

25. Pennington DW, Swienckowski JJ, Lutes WB, Drake GN. Unicompartmental knee arthroplasty in patients sixty years of age or younger. J Bone Joint Surg Am. 2003;85:1968-73.

26. Kim SJ, Postigo R, Koo S, Kim JH. Causes of revision following Oxford phase 3 unicompartmental knee arthroplasty. Knee Surg Sports Traumatol Arthrosc. 2014;22:1895-901.

27. Song MH, Kim BH, Ahn SJ, Yoo SH, Lee MS. Early complications after minimally invasive mobile-bearing medial unicompartmental knee arthroplasty. J Arthroplasty. 2009;24:1281-4.

28. Lim HC, Bae JH, Song SH, Kim SJ. Oxford phase 3 unicompartmental knee replacement in Korean patients. J Bone Joint Surg Br. 2012;94:1071-6.

29. Koh IJ, Kim JH, Jang SW, Kim MS, Kim C, In Y. Are the Oxford $\left(^{\oplus}\right)$ medial unicompartmental knee arthroplasty new instruments reducing the bearing dislocation risk while improving components relationships?: a case control study. Orthop Traumatol Surg Res. 2016;102:183-7. 